EPV170/\#68

\section{ONCOPHONE 20B: THE PATIENTS PERCEPTION OF TELEMEDICINE DURING FOLLOW UP VISITS FOR GYNECOLOGICAL CANCERS IN COVID-19 PANDEMIC}

${ }^{1} \mathrm{M}$ Mitidieri*, ${ }^{1} \mathrm{MG}$ Baù, ${ }^{2} \mathrm{~A}$ Surace, ${ }^{2} \mathrm{~F}$ Gallo, ${ }^{1} \mathrm{C}$ Anatrone, ${ }^{1} \mathrm{~A}$ Mondino, ${ }^{1} \mathrm{~S}$ Spallina, ${ }^{2} \mathrm{M}$ Carosso, ${ }^{2} \mathrm{P}$ Tondo, ${ }^{2} \mathrm{~S}$ Gemmiti, ${ }^{2} \mathrm{~L}$ Mosino, ${ }^{2} \mathrm{C}$ Benedetto, ${ }^{1} \mathrm{~S}$ Danese, ${ }^{1} \mathrm{E}$ Picardo. ${ }^{1} \mathrm{AOU}$ Citta della Salute e della Scienza di torino, Department of Gynecology and Obstetrics Sant'anna Hospital, Torino, Italy; ${ }^{2}$ University of Turin, Surgical Sciences, Torino, Italy

\subsection{6/ijgc-2021-IGCS.240}

Objectives The spread of COVID-19 pandemic changed the approach in the management of neoplasms. Telemedicine was one of the tools we experienced to maintain the continuity of care for the patients. Our goal is to evaluate the impact of telemedicine in patients management during follow up visits and its emotional impact.

Methods We enrolled 79 women with gynecological cancer. SUTAQ questionnaire was used to highlight patients perception about telemedicine. The questionnaire consists of 22 items divided into different subscales: 'Enhanced care'(EC), 'Satisfaction' (ST), 'Privacy and Discomfort'(PD), 'Care personnel concerns' (CPC), ' Increased accessibility' (IA) and ' Telemedicine as a Substitution' (TMS) scales

Results Enrolled women had a mean age of 55 years (35 women $\leq 55$ years and 44 women $\geq 55$ years). The majority of them $(61.54 \% ; n=48)$ achieved a high school diploma or higher while $(n=30)$ had a low educational level (middle school or lower); 87.3\% $(n=69)$ were employed and $70.89 \%$ $(n=56)$ lived with their partner. Younger women had a better perception towards telemedicine for TMS (mean=3.68) compared to older ones $($ mean $=3.05)$. The difference was statistically significant $(p=0.025)$. The PD subscale was in favor of higher educated women (mean=2.57) compared to lower educated ones $(m e a n=3.28 ; \mathrm{p}=0.042)$. No significant differences were observed between intensive and non intensive treatment. EC, ST, IA, and PD reached good responsiveness towards telemedicine, irrespectively of care level.

Conclusions Telemedicine has been a well-evaluated tool, not only among younger and higher educated women but even by women needing intensive care.

\section{EPV170a/\#392 EVALUATION OF HYSTEROSCOPY PRACTICE IN TIME OF COVID 19 PANDEMIC: A MULTICENTER EXPERIENCE}

${ }^{1} S$ Khedhri, ${ }^{2} \mathrm{MA}$ Ferjaoui*, ${ }^{3} \mathrm{R}$ Arfaoui, ${ }^{4} \mathrm{Y}$ Berrazega, ${ }^{5} \mathrm{MA}$ Hannachi, ${ }^{1} \mathrm{~A}$ Bellasoued, ${ }^{1} \mathrm{M}$ Malek, ${ }^{1} \mathrm{~K}$ Neji. ${ }^{1}$ Tunis Maternity Center, Department B of Gynecologic Surgery, Tunis, Tunisia; ${ }^{2}$ Tunis maternity center, Department B of Gynecologic Surgery, Tunis, Tunisia; ${ }^{3}$ Tunis military hospital, Department of Gynecology and Obstetrics, Tunis, Tunisia; ${ }^{4}$ University hospital Abderrahmen Mami Ariana, Medical Oncology, Tunis, Tunisia; ${ }^{5}$ Maternity and neonatology center of Tunis, B, Ben Arous, Tunisia

\subsection{6/ijgc-2021-IGCS.241}

Objectives The aims of our study were to assess different indications of office hysteroscopy and to evaluate the efficiency of this examination to diagnose uterine abnormalities in time of Covid 19 pandemic.

Methods This is a prospective and descriptive study from March 2020 to November 2020. 54 patients were enrolled in our study. They presented an abnormal uterine bleeding. The indication of hysteroscopy was discussed to know if deferring the procedure could have an impact on patient's condition and outcome. If hysteroscopy was indicated, preference was given to in-office procedure. In case of failure, patients underwent a hysteroscopy in an operating theatre under general or regional anesthesia. All hysteroscopies were performed under specific safety protocol.

Results 54 patients were enrolled in our study. office hysteroscopy was performed in all cases. The indication was related to a recurrent abnormal uterine bleeding complicated with anemia or associated to a thick endmetrium in post menopausal women. In four cases, office hysteroscopy was performed in infertile women. The failure rate of in-office hysteroscopy was $7.4 \%$. In 16 cases an operative hysteroscopy was performed in an operating room under anesthesia.Two cases of complex endometrial hyperplasia and one case of endometrial carcinoma were diagnosed. No cases of Covid 19 infection have been reported.

Conclusions office hysteroscopy is an efficient and safe examination to manage abnormal uterine bleeding in time of Covid 19 pandemic.

\section{EPV171/\#106 THE ATTRIBUTIVE VALUE OF COMPREHENSIVE SURGICAL STAGING IN CLINICALLY EARLY-STAGE EPITHELIAL OVARIAN CARCINOMA: A SYSTEMATIC REVIEW AND META-ANALYSIS}

${ }^{1} \mathrm{R}$ Van De Vorst ${ }^{*},{ }^{1} \mathrm{~J}$ Hoogendam, ${ }^{2} \mathrm{M}$ Van Der $\mathrm{Aa},{ }^{3} \mathrm{P}$ Witteveen, ${ }^{1} \mathrm{C}$ Gerestein, ${ }^{1} \mathrm{R}$ Zweemer. ${ }^{1}$ University Medical Center Utrecht, Imaging and Oncology, Utrecht, Netherlands; ${ }^{2}$ Netherlands Comprehensive Cancer Organization, Research, Utrecht, Netherlands; ${ }^{3}$ University Medical Center Utrecht, Medical Oncology, Utrecht, Netherlands

\subsection{6/ijgc-2021-IGCS.242}

Objectives To quantify tumor positivity and upstaging rates for all staging surgery steps in EOC patients. Differences between subgroups based on their clinical and histological characteristics are explored.

Methods A systematic search using synonyms of 'ovarian cancer', 'neoplasm staging', and 'neoplasm metastasis' was conducted in PubMed, Embase, and the Cochrane Library. Metaanalysis was performed on 23 included studies, comprising 5194 clinical stage I or II EOC patients who underwent comprehensive surgical staging. Studies were assessed using the Newcastle-Ottawa Scale risk-of-bias tool. Pooled proportions and $95 \%$ confidence intervals were calculated using an inverse variance weighted random-effects model.

Results Overall upstaging rate of clinically early-stage EOC patients was $18.7 \%$ (95\%CI: 14.1-23.4\%). Serous histology or high grade EOC showed the highest upstaging rate at $35.3 \%$ (95\%CI: $21.8-48.7 \%)$ and $40.9 \%$ (95\%CI: $35.6-46.2 \%)$. Lymph node involvement resulted in an upstaging rate of $8.7 \%$ (95\%CI: 6.2-11.3\%). Tumor was identified in uterus, cytology, peritoneal biopsies, omentum and appendix in $6.2 \%$ (95\%CI: $1.8-10.7 \%), \quad 18.4 \% \quad$ (95\%CI: $13.8-22.9 \%), \quad 9.7 \%$ (95\%CI: $3.8-15.6 \%), \quad 5.2 \% \quad(95 \% \mathrm{CI}: \quad 1.7-8.8 \%)$ and $3.6 \%$ (95\%CI: $0.0-7.5 \%)$ of EOC patients. The corresponding upstaging rates were 5.9\% (95\%CI: 1.4-10.4\%), 8.5\% (95\% CI: $1.8-15.2 \%), \quad 3.5 \%$ (95\%CI: $1.0-6.0 \%), 3.9 \% \quad(95 \% \mathrm{CI}:$ $1.4-6.3 \%)$ and $1.6 \%$ (95\%CI: $0.0-3.4 \%)$, respectively.

Conclusions The attributive value of comprehensive surgical staging in clinically early-stage EOC patients remains substantial, particularly in serous and high grade tumors. 